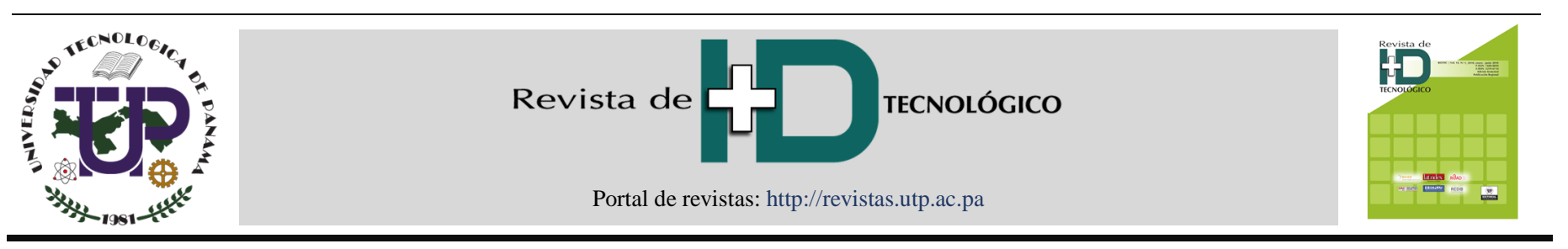

\title{
Efecto de los métodos de cocción por fritura en las propiedades fisicoquímicas y sensoriales de chips de taro (Colocasia esculenta)
}

\section{Effect of frying methods in the physicochemical and sensorial properties of taro chips (Colocasia esculenta)}

\author{
Melissa Chong ${ }^{1 *}$, Gina Mazzitelli ${ }^{1}$, Rosa Quintero ${ }^{2}$ \\ ${ }^{1}$ Licenciatura de Ingeniería en Alimentos - Facultad de Ciencias y Tecnología, Universidad Tecnológica de Panamá, Panamá \\ ${ }^{2}$ Grupo Ciencia y Tecnología Innovadora de Alimentos - Facultad de Ciencias y Tecnológica, Universidad Tecnológica de Panamá, Panamá \\ *Autor de correspondencia: melissachong1628@gmail.com
}

\begin{abstract}
RESUMEN- Taro (Colocasia esculenta) es un tubérculo comestible cultivados en regiones tropicales y subtropicales del mundo, incluyendo África, Asia, Hawái, América Central, América del Sur, el Caribe y México. En este estudio se elaboraron chips de taro mediante dos métodos de cocción, freído convencional y freído con aire forzado. El objetivo de este trabajo fue determinar el efecto de ambos métodos sobre las características fisicoquímicas y sensoriales de los chips elaborados. Al producto obtenido se realizaron pruebas de humedad, ceniza, lípido, proteínas, fibras y evaluación sensorial, además se determinó el contenido de oxalato en taro crudo y los chips elaborados. El chip elaborado por el método convencional mostró una mayor crujencia (208.95 gf), alto contenido de grasa (32.53\%), menor concentración de oxalato $0.40 \mathrm{mg} / 100 \mathrm{~g}$ muestra y mayor preferencia (87\%) por parte del consumidor en comparación con el chip elaborado con la freidora de aire (21.53\% de grasa, $2.48 \mathrm{mg}$ oxalato/100 g muestra y dureza 40908 gf). En conclusión, se logró elaborar chips de taro, un tubérculo poco conocido y comercializado, con característica nutricionales importante como es el contenido de carbohidratos. Por otra parte, el método de freído por aire puede ser una alternativa saludable que reduce significativamente el contenido de grasa y presenta características sensoriales aceptables en productos fritos.
\end{abstract}

Palabras clave-Colocasia esculenta, crujiente, freidora de aire, oxalatos, taro.

ABSTRACT - Taro (Colocasia esculenta) is an edible root grown in tropical and subtropical regions of the world, including Africa, Asia, Hawaii, Central America, South America, the Caribbean and Mexico. In this study, taro chips were processed by two methods of cooking, conventional frying and air frying. The objective of this work was to determine the effect of both methods on the physicochemical and sensorial characteristics of the processed chips. The product obtained was tested for moisture, ash, lipid, protein, fibers and sensorial evaluation, in addition, the oxalate content both, in raw taro and the processed chips were determined. The chip by the conventional process showed greater crispy, high fat content (32.53\%), lower oxalate concentration $(0.40 \mathrm{mg} / 100 \mathrm{~g}$ sample) and higher preference by the consumer, compared to the chip air fried process fat contents (fat contents $21.53 \%$, oxalates contents $2.48 \mathrm{mg} / 100 \mathrm{~g}$ sample and hardness $40908 \mathrm{gf}$ ). In conclusion, it was possible to process taro chips, a not well known and commercialized root with important nutritional characteristics, such as carbohydrate content. On the other hand, the air frying could be an alternative method to prepare healthy food, reducing fat content and with acceptable sensorial attributes.

Keywords-Colocasia esculenta, crispy, air fryer, oxalates, taro.

\section{Introducción}

El taro es el tubérculo comestible de la planta Colocasia esculenta, y es un importante ingrediente en muchas comidas de Asia y las Islas de Pacífico [1]. Actualmente es uno de los principales cultivos en regiones tropicales y subtropicales del mundo, incluyendo África Occidental, África Central, Asia, Hawái, América Central, América del Sur, el Caribe y México; su cultivo ha aumentado pero su uso ha sido limitado debido a su corta vida postcosecha [2]. El tubérculo es de rápido desarrollo vegetativo, fácil de cultivar, muy resistente al ataque de plagas y enfermedades [3]. La planta pertenece a la familia de las Aráceas, y producen en su base un cormo alargado (tubérculo) de forma ovoide, que es la parte comestible, aunque en algunas culturas también consumen las hojas [1]. La cáscara es de color café y la pulpa es blanca con vetas de color rosadas a lilas [4].

El tubérculo es una excelente fuente de energía, por esta particularidad son rápidamente digeribles tanto para el hombre como para los animales. Recientemente el taro ha adquirido gran importancia, se considera como

Citación: M. Chong, G. Mazzitelli y R. Quintero., "Efecto de los métodos de fritura por inmersión y no inmersión en las propiedades fisicoquímicas y sensoriales de chips de taro (Colocasia esculenta)", Revista de I+D Tecnológico, vol. 15, no. 1, pp. (30-37), 2019.

Tipo de artículo: Original. Recibido: 27 de julio de 2018. Recibido con correcciones: 22 de agosto de 2018. Aceptado: 19 de noviembre de 2018.

DOI https://doi.org/10.33412/idt.v15.1.2095

Copyright: 2019 M. Chong, G. Mazzitelli y R. Quintero. This is an open access article under the CC BY-NC-SA 4.0 license (https://creativecommons.org/licenses/bync-sa/4.0/). 
materia prima de gran potencial por su alto contenido de carbohidratos, principalmente de almidón (700-800 g/kg base seca) [3]. Estos tubérculos presentan gran contenido de calcio, magnesio, potasio, zinc, hierro, proteína y aminoácidos que otras raíces y tubérculos tropicales [5]. Puede reemplazar el uso de materias primas convencionales como maíz, plátano, yuca y papa, utilizando tecnologías sencillas y aprovechando este recurso agrícola que sirve para la elaboración de diversos productos tipo snack [6].

Estudios han reportado que las variedades de taro contienen una cantidad significativa de oxalato que se distribuyen en toda la planta y se encuentran de forma soluble como los oxalatos de potasio, sodio y amonio, y de forma insoluble como calcio. El oxalato de calcio se presenta en como cristales en forma de finas agujas y causan un sabor acre y pueden causar hinchazón e irritación en los labios, boca y garganta si se comen crudos [7]. La presencia de oxalato en los alimentos se ha visto implicada en la reducción de la biodisponibilidad del calcio, esto conlleva a que el consumo de cantidades considerables de oxalato puede ser fatal para el organismo porque puede promover la formación de depósitos de oxalato de calcio en los tejidos y órganos del cuerpo [8]. Es el principal componente de los cálculos renales, por lo que las personas con este problema de salud deben tener una dieta controlada para alimentos que contienen oxalato [9]. La American Dietetic Association recomienda una ingesta diaria máxima de 40-50 mg de oxalato [10].

Los procesos de remojo, cocción y fermentación pueden reducir el contenido de oxalato en el taro hasta niveles seguros [11].

Tradicionalmente los chips se elaboran por freído convencional a escala doméstica e industrial. Es un proceso de deshidratación que requiere una transferencia rápida de calor y masa cuando el alimento se sumerge en aceite caliente $\left(150-190{ }^{\circ} \mathrm{C}\right)$, se produce una textura crujiente, olores, colores y sabores agradables [12]. Sin embargo, está la preocupación de los consumidores por los problemas de salud como la obesidad, hipertensión, diabetes y cáncer, asociados al frecuente consumo de este tipo de producto, con alto contenido de grasa, calorías y formación de sustancias tóxicas como la acrilamida.

En los últimos años se ha incrementado los estudios en nuevas alternativas tecnológicas en el procesamiento de alimentos que reduzca el contenido de grasa

RIDTEC | Vol. 15, n. ${ }^{\circ}$ I, enero - junio 2019. manteniendo la calidad nutricional y sensorial de los mismos. El freído por aire forzado es un método económico y se encuentran equipos de uso domésticos comercialmente disponible a nivel mundial. En este proceso, el alimento frito se obtiene por contacto directo del producto y una emulsión externa de gotas de aceite en aire caliente dentro de una cámara que está en movimiento constante [13] [14]. Imita la deshidratación y la formación de la corteza típica de los alimentos fritos de la manera convencional. Estudios han demostrado que el contenido de lípidos en los productos elaborados por este método es significativamente más bajo, la formación de acrilamina se reduce y los atributos sensoriales pueden ser similares a los fritos por inmersión en aceite [15] [16].

Por lo antes expuesto, el objetivo de este estudio fue evaluar la viabilidad de elaborar chips de taro y determinar el efecto de los métodos de freído convencional y con aire forzado en las características fisicoquímicas y atributos sensoriales.

\section{Materiales y métodos}

Los tubérculos de taro se obtuvieron frescos en un supermercado de la localidad, cuyo cultivo procedía de la población de Chilibre, provincia de Panamá. Se seleccionaron muestras pequeñas con diámetros entre 5 a $6 \mathrm{~cm}$. Para su conservación se envolvió con filme plástico PVC para alimentos y se almacenó a $5{ }^{\circ} \mathrm{C}$ para su posterior análisis.

\subsection{Elaboración de los chips}

Los tubérculos se lavaron, pelaron y rebanaron con un pelador de vegetales para conseguir un grosor de 1 a 2 $\mathrm{mm}$.

\subsubsection{Método de cocción: freído en aceite}

El freído se realizó de forma convencional por inmersión en aceite en una freidora de acero inoxidable de 2 litros de capacidad. Se utilizó 1 litro de aceite vegetal y se frieron en porciones de $35 \mathrm{~g}$ a $180{ }^{\circ} \mathrm{C}$ por $1 \mathrm{~min}$. Posteriormente se colocaron sobre una malla que permitiera el escurrido del exceso de aceite y se guardaron en bolsas de polietileno con cierre tipo ziploc para su posterior análisis.

\subsubsection{Método de cocción: freído por aire}

A las rebanadas se les aplicó una ligera capa del mismo aceite por ambos lados, con una brocha de silicona. Se utilizó una freidora de aire marca RCA 
modelo RCFR10 con $2.8 \mathrm{~L}$ de capacidad con un poder de $1200 \mathrm{~W}$. Se coloraron en porciones de 50g distribuidas equitativamente por toda la malla. Se realizó su cocción durante 5 min a $180{ }^{\circ} \mathrm{C}$ por cada lado, se dejó enfriar a temperatura ambiente por $10 \mathrm{~min}$ y se guardaron en bolsas de polietileno con cierre tipo ziploc para su posterior análisis.

\subsection{Caracterización Fisicoquímica}

El contenido de humedad se determinó por medio de un analizador de humedad precisa modelo XM-50. El contenido de grasa, proteína (se calculó en base al contenido de nitrógeno y un factor 6.25), fibra y ceniza se determinaron de acuerdo con los métodos oficiales de AOAC [17] [18]. El contenido de carbohidrato total se determinó por diferencia, el porcentaje de carbohidratos $=100-(\%$ humedad $+\%$ grasa $+\%$ proteína $+\%$ fibra + $\%$ ceniza) [7].

\subsection{Determinación del contenido de oxalato}

Se determinó el contenido de oxalato en el cormo crudo y en los dos chips según la metodología de Kumoro et al. [7]. El procedimiento implica tres pasos: digestión, precipitación del oxalato y titulación con permanganato de potasio. El contenido de oxalato se calculó usando la fórmula:

$$
\frac{\mathrm{mg} \text { de oxalato }}{100 \mathrm{~g} \text { de muestra }}=\frac{\mathrm{T} \times \mathrm{V}_{\mathrm{me}} \times \mathrm{DF} \times 10^{5}}{\mathrm{ME} \times \mathrm{mf}}
$$

donde $T$ es el volumen consumido de $\mathrm{KMnO}_{4}(\mathrm{~mL}), V_{\text {me }}$ es el volumen-equivalente de masa $\left(1 \mathrm{~mL}\right.$ de $\mathrm{KMnO}_{4}$ $0.05 \mathrm{M}$ es equivalente a $0.00225 \mathrm{~g}$ de ácido oxálico anhidro), $D F$ es el factor de dilución VTA (2, donde VT es el volumen total de filtrado (300 ml) y A es la alícuota utilizada $(150 \mathrm{ml})), M E$ es el equivalente molar de $\mathrm{KMnO}_{4}$ en oxalato en la reacción redox (5) y $m f$ es la masa de muestra utilizada.

\subsection{Evaluación sensorial}

Ambas muestras fueron evaluadas por 40 jueces no entrenados usando las pruebas de preferencia y comparación pareada simple. En la primera prueba se presentó las muestras de chips en forma aleatoria y debidamente codificados a los panelistas, después de probar cada uno se indicó el código de su preferencia en el formato entregado. La segunda prueba se realizó de la misma forma con la diferencia que se evaluó el atributo de crujencia.

\subsubsection{Perfil sensorial}

Se realizó la prueba a 23 jueces semientrenados para determinar el perfil sensorial general. La prueba consistió en presentar ambos chips codificados, a las cuales se le evaluó la intensidad de los atributos de olor (aceite), sabor (salado y aceite), crujencia y apariencia general (aceite en la superficie) mediante escala de intervalo de 5 puntos ( 0 baja intensidad y 4 mayor intensidad)

\subsubsection{Análisis de textura}

La dureza de ambos chips fue analizada con un texturómetro Stable Micro Systems modelo TA.XT.plus con una sonda $\mathrm{P} / 2$ cilíndrica de $2 \mathrm{~mm}$ diámetro de acero inoxidable. Se realizaron 10 réplicas, seleccionando previamente los chips más homogéneos [19]. Se realizó un ensayo de compresión, fijando una distancia de avance $3.0 \mathrm{~mm}$ y fuerza de impulso de 5.0 gf. Tanto la velocidad de pre-ensayo como la de ensayo fueron de $1.00 \mathrm{~mm} / \mathrm{s}$.

\subsection{Análisis estadístico}

Análisis fisicoquímicos: se realizaron por triplicado presentándose como valores promedios con su desviación estándar, con un nivel de confianza de $95 \%$. Evaluación sensorial: los datos fueron analizados mediante ANOVA mediante la hoja de cálculo Excel 2016 y se aplicó prueba de Duncan se utilizó para establecer las diferencias significativas $(\mathrm{p}<0.05)$ entre las muestras

\section{Resultados y discusión \\ 3.1 Caracterización fisicoquímica}

La humedad y el contenido de aceite son características importantes en los productos fritos. Durante el proceso de fritura el contenido de agua se pierde casi en su totalidad. En la tabla 1 se presentan la composición del taro crudo según la literatura [20], donde el contenido de humedad es de $70.64 \%$, y en la tabla 2 se muestra la composición de los chips, y se observa que la humedad en los chips fritos por inmersión $(0.62 \%)$ fue mucho más bajo que en los chips de freidora de aire $(2.78 \%)$. En cuanto al contenido de grasa, los chips obtenidos por método convencional presentaron un contenido de $31.53 \%$, mientras que el freído por aire $21.53 \%$, lo cual presenta una reducción del $32 \%$. 
Tabla 1. Composición proximal de los cormos de taro crudo por cada $100 \mathrm{~g}$ de porción comestible

\begin{tabular}{cc}
\hline Componente & Valor $(\mathbf{g})$ \\
\hline Humedad & 70.64 \\
\hline Lípidos & 0.20 \\
\hline Proteína & 1.50 \\
\hline Carbohidratos & 26.46 \\
\hline Fibra cruda & 4.10 \\
\hline Ceniza & 1.20
\end{tabular}

Fuente: INCAP. Tabla de composición de los alimentos de Centroamérica.

Tabla 2. Composición proximal de los chips de taro por cada $100 \mathrm{~g}$

\begin{tabular}{|c|c|c|}
\hline \multirow{2}{*}{ Componente } & \multicolumn{2}{|c|}{ Tipo de chip } \\
\cline { 2 - 3 } & $\begin{array}{c}\text { Inmersión en } \\
\text { aceite }\end{array}$ & $\begin{array}{c}\text { Freidora de aire } \\
\text { forzado }\end{array}$ \\
\hline Humedad (g) & $0.62 \pm 0.12^{\mathrm{a}}$ & $2.78 \pm 0.12^{\mathrm{b}}$ \\
\hline Grasa $(\mathbf{g})$ & $31.53 \pm 0.28^{\mathrm{a}}$ & $21.53 \pm 1.45^{\mathrm{b}}$ \\
\hline Proteína $(\mathbf{g})$ & $5.05 \pm 0.88^{\mathrm{a}}$ & $6.66 \pm 0.90^{\mathrm{a}}$ \\
\hline Carbohidrato* $(\mathbf{g})^{\mathrm{a}}$ & 55.86 & 60.03 \\
\hline Fibra cruda $(\mathbf{g})$ & $2.98 \pm 0.32^{\mathrm{a}}$ & $3.89 \pm 0.03^{\mathrm{a}}$ \\
\hline Ceniza $(\mathbf{g})$ & $4.17 \pm 0.20^{\mathrm{a}}$ & $5.24 \pm 0.43^{\mathrm{a}}$ \\
\hline
\end{tabular}

Lo resultados están expresado como valor promedio $(n=3)$ con desviación estándar.

*Los valores de carbohidrato se determinaron por diferencia.

Letras iguales en la misma fila indica que no hay diferencia significativa entre las muestras.

Se obtuvieron resultados similares en estudios con papas fritas por inmersión en aceite, donde se observó que estas absorben cantidades elevadas de aceite, y el contenido de grasa es alrededor de $30 \%$ y fritas por aire forzado el contenido graso alrededor de $15 \%$ [15] [13]. La alta temperatura del aceite favorece la conducción de calor, el agua migra del alimento al aceite y se evapora al contacto [21]. El aumento de tiempo de cocción incrementa la evaporación del agua favoreciendo la porosidad y formación de espacio vacío que puede ser reemplazado por el aceite [22].

El contenido de carbohidrato y proteína es similar en ambos chips, y la pérdida respecto al taro crudo es poca. En estudios realizados se sostiene que el cambio de color de los chips después de la fritura se debe principalmente al contenido de azúcares reductores presente en las raíces y tubérculos. Si este contenido es bajo, se obtendrán chips levemente dorados de buena calidad, sin embargo, un alto contenido en el producto provocará una coloración marrón oscura, debido a reacciones de oscurecimiento no enzimática tales como la de Maillard y cambios estructurales acelerados por las altas temperaturas del aceite de fritura [23]. En la figura 1 se observan los dos chips, visualmente son muy similares en color y forma, y presentan una coloración amarillenta leve, sin llegar al tostado.

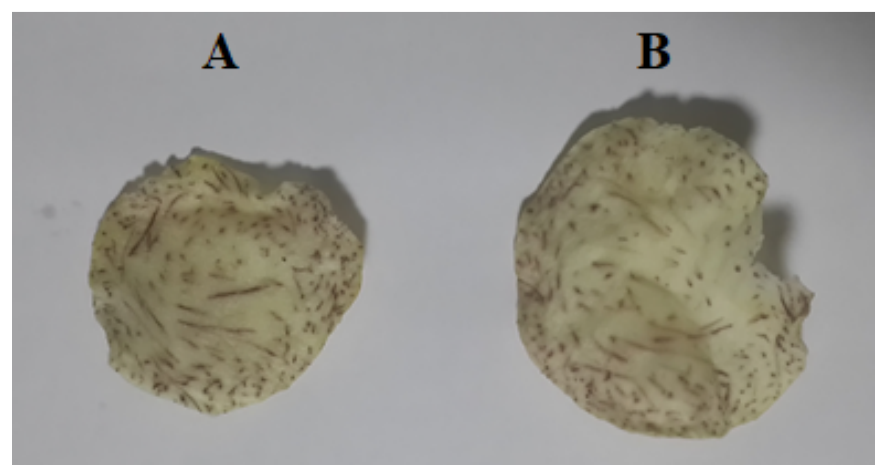

Figura 1. Chips de taro: (A) chip por inmersión en aceite, (B) chip elaborado en freidora de aire.

El contenido de fibra y ceniza no varían en gran medida entre ambos chips, y permanecen respecto al taro crudo. El efecto del contenido de fibra se puede apreciar en la textura, favoreciendo la crujencia. Estudios realizados demuestran que el contenido de fibra aumenta con la edad de cultivo, si el contenido de fibras es muy alto se obtendrán chips demasiado duros [24].

\subsection{Contenido de oxalato}

En la tabla 3 se presentan el contenido de oxalato para el taro crudo y los dos chips. Diversos estudios han reportado el contenido de oxalato para taro crudo fresco $65 \mathrm{mg} / 100 \mathrm{~g}$ [25], 367-710 mg/100g y $780 \mathrm{mg} / 100 \mathrm{~g}$ para variedades provenientes de África, además depende de factores ambientales, sequía [26]. El contenido de oxalato en taro crudo investigado en este estudio fue de $0.30 \mathrm{mg} / 100 \mathrm{~g}$ (base húmeda), y no concuerda con los valores reportados. Esto puede atribuirse a pérdidas durante el procesamiento de las muestras. El método se basa en la precipitación del oxalato como oxalato de calcio y tiene algunas limitaciones en cuanto a sensibilidad, precisión y tiempo requerido para su desarrollo [27]. Por otra parte, cabe recalcar que en este estudio se utilizaron taros pequeños, que se cosecharon en una etapa temprana, mientras más tiempo tarde en 
cosecharse mayor tamaño adquirirá y la concentración de oxalato aumenta con el tamaño [28].

Tabla 3. Contenido de oxalato por cada $100 \mathrm{~g}$ de muestra

\begin{tabular}{|c|c|}
\hline Muestra & mg de oxalato \\
\hline Taro crudo (base seca) & $1.03 \pm 0.16^{\mathrm{a}}$ \\
\hline Chip de freidora convencional $^{\mathrm{b}}$ & $0.40 \pm 0.05^{\mathrm{b}}$ \\
\hline Chip de freidora de aire $^{\mathrm{c}}$ & $2.48 \pm 0.22^{\mathrm{c}}$ \\
\hline
\end{tabular}

Lo resultados están expresado como valor promedio $(n=3)$ con desviación estándar.

Letras diferentes en la misma columna indica que hay diferencia significativa entre las muestras.

Los niveles de oxalatos son altos en la cáscara del taro y va disminuyendo hacia la parte comestible. Se espera que los procesos de cocción reduzcan el contenido de oxalato. Sin embargo, solo se observa reducción en el chip de inmersión, lo que se puede atribuir a la descomposición térmica del oxalato en carbonato y monóxido de carbono debido al gran calor generado en este proceso [7]. El proceso de cocción de la freidora de aire es similar al horneado, y se ha reportado que hornear el taro tiene el efecto de concentrar el oxalato en la matriz del alimento debido a la pérdida de agua [1], pero en contraparte también, el secado del taro disminuye la concentración de oxalato en un $50 \%$, debido a la degradación térmica de oxalato mencionada anteriormente [11].

Por lo antes mencionado, el taro debe pasar por un proceso de cocción para inactivar los pequeños cristales de oxalato de calcio que causan irritación en la boca, lo que desfavorece las propiedades sensoriales y disminuye la toxicidad [29].

El método reportado más eficiente para reducir los niveles de oxalato en el taro es por lixiviación durante el hervido, disminuye en un $56 \%$ si se hierve por $40 \mathrm{~min}$ [1]. Similarmente en estudios sobre la oca (Oxalis tuberosa) demostraron que el hervido disminuye significativamente la concentración de oxalato en todo el tubérculo [30].

\subsection{Evaluación sensorial}

En los resultados de las pruebas sensoriales aplicadas se reportan en la tabla 4. En la prueba de preferencia y se observa que 35 panelista prefieren el chip elaborado por el método de freído convencional, lo que representa un
$87 \%$ en comparación con el $13 \%$ obtenido por el método de freído por aire forzado. Por otra parte, la prueba de comparación pareada aplicada indicó que el $80 \%$ de los panelistas detectan mayor crujencia en el chip de freído convencional en comparación con el otro chip.

Tabla 4. Resultados de las pruebas sensoriales

\begin{tabular}{|c|c|c|}
\hline Tipo de Chip & Preferencia & $\begin{array}{c}\text { Comparación } \\
\text { pareada simple }\end{array}$ \\
\hline Freidora convencional & 35 & 32 \\
\hline Freidora de aire & 5 & 8 \\
\hline
\end{tabular}

Fuente: cantidad de panelista 40.

En la tabla 5 se presentan las puntuaciones medias obtenida del perfil sensorial referente a los atributos de olor a aceite, crujencia, sabor salado y graso. En todos los parámetros evaluados se observó los mayores valores para el chip elaborado por inmersión en aceite, similares resultados se obtuvieron en papas frita elaboradas con ambos métodos [13]. La presencia de aceite en el chip de freído convencional es muy perceptible, y crea una diferencia global con el chip de freído por aire. Se ha reportado que la mayor parte del contenido de aceite de los productos fritos se encuentra en la superficie, y que es principalmente adquirido durante el período de enfriamiento y no durante el freído como se espera. Se presume que, durante el freído, la evaporación del agua del producto es tan vigoroso que genera una barrera para evitar la entrada de aceite hacia la estructura porosa del alimento y como consecuencia, la absorción de aceite se ve limitada durante la mayor parte del período de inmersión. Como resultado, la absorción de aceite va a depender de la succión en la corteza y del drenaje una vez que se retira el producto del aceite [31].

Tabla 5. Parámetros sensoriales de los chips

\begin{tabular}{lcc}
\hline \multirow{2}{*}{ Parámetro } & \multicolumn{2}{c}{ Tipo de Chip } \\
\cline { 2 - 3 } & $\begin{array}{c}\text { Freidora } \\
\text { convencional }\end{array}$ & Freidora de aire \\
\hline $\begin{array}{l}\text { Aceite en la } \\
\text { superficie }\end{array}$ & $2.78 \pm 1.13^{\mathrm{a}}$ & $0.74 \pm 1.10^{\mathrm{b}}$ \\
\hline Olor a aceite & $2.89 \pm 0.94^{\mathrm{a}}$ & $1.00 \pm 0.88^{\mathrm{b}}$ \\
\hline Sabor salado & $2.27 \pm 0.88^{\mathrm{a}}$ & $0.82 \pm 0.73^{\mathrm{b}}$ \\
\hline Sabor a aceite & $2.68 \pm 1.17^{\mathrm{a}}$ & $0.95 \pm 0.84^{\mathrm{b}}$ \\
\hline Crujiente & $3.55 \pm 0.60^{\mathrm{a}}$ & $2.20 \pm 1.06^{\mathrm{b}}$ \\
\hline
\end{tabular}


Lo resultados están expresado como valor promedio $(n=23)$ con desviación estándar.

Letras diferentes en la misma fila indica que hay diferencia significativa entre las muestras.

En el parámetro del sabor salado se observó diferencia significativa, a pesar de que se agregó sal en la misma proporción. La mayor percepción del sabor salado fue en el chip de freido convencional. Esto puede ser atribuido a que el contenido graso del chip aumenta la percepción del sabor salado. Se ha encontrado que reducir el contenido graso disminuye las sensaciones gustativas básicas. Estudios realizados donde redujeron el contenido graso en queso, aderezo para ensalada y mayonesa, demostraron que la percepción del sabor salado fue con menor intensidad en comparación con los alimentos con contenido graso completo [32].

\subsection{Análisis de textura}

En la industria de los snacks fritos se busca una textura crujiente en los productos, que es la principal propiedad que los distingue. La dureza se define como la fuerza máxima requerida para que se dé la ruptura de la muestra, y se relaciona con cuan crujiente está el chip [16]. Una menor dureza indica que un alimento es más crujiente. Los resultados del análisis de los datos generados por el texturómetro se presentan en la tabla 6 y demostraron que la dureza del chip de inmersión es significativamente menor $(\mathrm{p}<0.05)$ que la del chip de freidora de aire, siendo el chip de inmersión el más crujiente. Estos los resultados son congruentes con los obtenidos en las pruebas apareada simple y perfil sensorial. Existe diferencia significativa en el crujiente de los chips por el método cocción por fritura aplicado

Tabla 6. Resultados de dureza obtenidos para los chips

\begin{tabular}{lc}
\hline \multicolumn{1}{c}{ Tipo de Chip } & Dureza $(\mathbf{g f})^{*}$ \\
\hline \multicolumn{1}{c}{ Inmersión en aceite } & $208.95 \pm 10.40^{\mathrm{a}}$ \\
\hline \multicolumn{1}{c}{ Freidora de aire } & $40908.81 \pm 487.05^{\mathrm{b}}$ \\
*gf = gramos fuerza. \\
*Los valores están expresados como valor medio \pm desviación \\
estándar (n=10). \\
$\begin{array}{l}\text { Letras diferentes en la misma } \\
\text { diferencia significativa. }\end{array}$
\end{tabular}

Otros factores que afectan el crujiente es la falta de uniformidad en el chip, la variabilidad en la humedad y la distribución heterogénea del almidón y otros compuestos que afecta a la microestructura de los alimentos después de la fritura. La variabilidad de las técnicas usadas, el manejo y almacenamiento de los chips antes de realizar las mediciones, son las principales variables que deben ser consideradas en los estudios de textura de este producto [19]. La textura depende mucho de la humedad del producto, ya que, al deshidratarse el almidón a altas temperaturas, este se retrograda y provoca un cambio en la estructura interna que se ve reflejado en una disminución de la fuerza de ruptura de las células [33].

\section{Conclusiones}

Se lograron elaborar chips de taro por los métodos de fritura convencional y por aire forzado. Los resultados mostraron mayores diferencias en el contenido de grasa, crujencia y preferencia por parte del panel sensorial. Los chips elaborados por freído convencional mostraron el $87 \%$ preferencia siendo los atributos de crujencia y sabor los de mayor puntuación. Por otra parte, presentaron un $31.53 \%$ de lípidos, menor contenido de oxalato $0.40 \mathrm{mg}$ $/ 100 \mathrm{~g}$ muestra y menor dureza 208.95 gf. En comparación al freído por aire $(21.53 \%$ de lipido, 4.48 mg oxalato/100 g muestra y 40908 gf de dureza).

Es necesario seguir realizando investigaciones con el método de freído con aire forzado, tecnología que permitirá obtener productos más saludables con propiedades nutricionales y sensoriales aceptables

\section{Agradecimiento}

Los autores agradecen a la Coordinación de Química, Departamento de Ciencias Naturales de la Facultad de Ciencias y Tecnología de la Universidad Tecnológica de Panamá, por el apoyo brindado en el desarrollo de este estudio, especialmente a la Dra. Indira Franco, Lcdo. Alejandrino Sevillano, Lcda. Johanna Rodríguez y Marialina Anria por su asesoramiento y asistencia técnica. Se agradece también a la Ing. Cindy Mayorga del Centro de Producción e Investigaciones Agroindustriales de la Universidad Tecnológica de Panamá, por su asistencia en los análisis de textura.

\section{Referencias}

[1] D.J. Catherwood, G.P. Savage, S.M. Mason, J.J.C. Scheffer and J.A. Douglas, "Oxalate content of cormels of Japanese taro (Colocasia esculenta (L.) Schott) and the effect of cooking," 
Journal of Food Composition and Analysis, vol. 20, pp. 147151,2007

[2] A. Torres, M. Durán y P. Montero, "Evaluación de las propiedades funcionales del almidón obtenido a partir de malanga (Colocasia esculenta)," Revista Ciencias e Ingeniería al Día, vol. 8, n², pp. 29-38, 2013.

[3] Q.W. Caicedo, B.R. Rodríguez y R.S. Valle, "Una reseña sobre el uso de tubérculos de papa china Colocasia esculenta conservados en forma de ensilaje para alimentar cerdos," Revista Electrónica de Veterinaria, vol. 15, n¹, 2013.

[4] K. Ríos, "Análisis comparativo de las propiedades físicoquímicas y nutrimentales de almidón obtenido a partir de dos especies de malanga (Colocasia antiquorum y Colocasia esculenta) cultivadas en el estado de Oaxaca," Tesis de licenciatura, Universidad Tecnológica de la Mixteca, Huajuapan de León, Oaxaca, México, 2014.

[5] C.A. Estrada, L.A. Bello, C.E. Martínez, J.L. Montañez, J. Jiménez y M.A. Vivar, "Producción enzimática de maltodextrinas a partir de almidón de malanga (Colocasia esculenta)," CyTA - Journal of Food, vol. 7, n³, pp. 233-241, 2009.

[6] A. Torres, P. Montero y M. Durán, "Propiedades fisicoquímicas, morfológicas y funcionales del almidón de malanga (Colocasia esculenta)," Revista Lasallista de Investigación, vol. 10, $\mathrm{n}^{\circ} 2,2013$.

[7] J.H. Bradbury and R.W. Nixon, "The Acridity of Raphides from the Edible Aroids," Journal of the Science of Food \& Agriculture, vol. 76, n4, pp. 608-616, 1998.

[8] A.C. Kumoro, R.D.A. Putri, C.S. Budiyati, D. Susetyo, Retnowati and Ratnawati, "Kinetics of calcium oxalate reduction in Taro (Colocasia esculenta) corm chips during treatments using baking soda solution," Procedia Chemistry, vol. 9, pp. 102-112, 2014.

[9] Quan-Yuan Ruan, Xue-Qin Zheng, Bai-Ling Chen, Yang Xiao, Xin-Xiang Peng, David W.M. and Leung, E-E Liu, "Determination of total oxalate contents of a great variety of foods commonly available in Southern China using an oxalate oxidase prepared from wheat bran," Journal of Food Composition and Analysis, vol. 32, pp. 6-11, 2013.

[10] Urolithiasis/urinary stones, ADA Nutrition Care Manual of the American Dietetic Association, Chicago, IL, USA, 2005.

[11] S. Sefa-Dedeh and E. K. Agyir-Sackey, "Chemical composition and the effect of processing on oxalate content of cocoyam Xanthosoma sagittifolium and Colocasia esculenta cormels," Food Chemistry, vol. 85, pp. 479-487, 2004.

[12] F. Pedreschi, "Frying of potatoes: physical, chemical, and microstructural changes," Drying Technology: an-International Journal, vol. 30, pp. 707-725, 2012.

[13] J. Tian, S. Chen, J. Shi, J. Chen, D. Liu, Y. Cai, Y. Ogawa, and $\mathrm{X}$. Ye, "Microstructure and digestibility of potato strips produced by conventional frying and air-frying: an in vitro study," Food Structure, vol. 14, pp. 30-35, 2017.

[14] S.K. Pankaj and K. M. Keener, "A review and research trends in alternate frying technologies", Current Opinion in Food Science, vol. 16, pp. 74-79, 2017
[15] S.M. Arafat, "Air frying a new technique for produce of healthy fried potato strips," Journal of Food and Nutrition Sciences, vol. 2, nº , pp. 200-206, 2014.

[16] B. Belkova, J. Hradecky, K. Hurkova, V. Forstova, L. Vaclavik and J. Hajslova, "Impact of vacuum frying on quality of potato crisps and frying oil," Food Chemistry, vol. 241, pp. 51-59, 2018.

[17] AOAC International, Official Methods of Analysis, $17^{\circ}$ ed., Gaithersburg, USA: Association of Official Analytical Chemists, 2000.

[18] AOAC International, Official Methods of Analysis, $30^{\circ}$ ed., Washington, USA: Association of Official Analytical Chemists, 1980.

[19] C. Castro, "Evaluación de las propiedades físico-químicas y sensoriales de la patata para fritura". Tesis de Maestría, Universidad de Burgos, Burgos, España, 2008.

[20] INCAP y OPS, Tabla de composición de los alimentos de Centromérica, 2da ed., 2007.

[21] M.D.R. Teruel, M. Gordon, M.B. Linares, M.D. Garrido, A. Ahromrit and K. Niranjan, "A comparative study of the characteristics of French fries produced by deep fat frying and air Frying," Journal of Food Science, vol, 80, n², pp. 349-358, 2015.

[22] Y. Zhang, T. Zhang, D. Fan, J. Li, L. Fan, “The description of oil absorption behavior of potato chips during the frying", LWT-Food Science and Technology, vol. 96, pp. 119-126, 2018.

[23] J. Hasbún, P. Esquivel, A. Brenes y I. Alfaro,” Propiedades físico-químicas y parámetros de calidad para uso industrial de cuatro variedades de papa," Agronomía Costarricense, vol. 33, nº 1 , pp.77-89, 2009.

[24] N.P. Durán, I. Osorio and M.A. Rojas,” Proposed methodology for the evaluation of the physicochemical characteristics of two varieties of cassava (Manihot esculenta Crantz), used as raw material for the preparation of fried chips," Scientia et Technica Año XVIII, vol. 18, n³, pp. 553-559, 2013.

[25] W. D. Holloway, M. E. Argall, W.T. Jealous, J.A. Lee and J.H. Bradbury, "Organic acids and calcium oxalate in tropical root crops," Journal of Agriculture and Food Chemistry, vol. 37, pp. 337-341, 1989.

[26] Organización de las Naciones Unidas para la Agricultura y la Alimentación, Raíces, tubérculos, plátanos y bananas en la nutrición humana. Roma, Italia: FAO, 1991, pp. 108.

[27] K. Judprasong, S. Charoenkiatkul, P. Sungpuag, K. Vasanachitt and Y. Nakjamanong, "Total and soluble oxalate contents in Thai vegetables, cereal grains and legume sedes and their changes after cooking," Journal of Food Composition and Analysis, vol. 19 n4, pp. 340-347, 2006.

[28] A. Montaldo, Cultivo de raíces y tubérculos tropicales. San José, Costa Rica: Instituto Interamericano de Cooperación para la Agricultura, 1991, pp. 59-62.

[29] Aboubakar, N.Y. Njintang, J. Scher and C.M.F. Mbofung, "Texture, microstructure and physicochemical characteristics of taro (Colocasia esculenta) as influenced by cooking 
conditions," Journal of Food Engineering, vol.91, pp. 373-379, 2009.

[30] P.B.E. Albihn and G.P. Savage, "The effect of cooking on the location and concentration of oxalate in three cultivars of New Zealand-grown oca (Oxalis tuberosa Mol)," Journal of the Science of Food and Agriculture, vol. 81, pp. 1027-1033, 2001

[31] V. Dueik and P. Bouchon, "Development of Healthy Low-Fat Snacks: Understanding the Mechanisms of Quality Changes During Atmospheric and Vacuum Frying," Food Reviews International, vol. 27, nº 4, pp.408-433, 2011.
[32] E. Kostyra and N. Baryłko-Pikielna. "The effect of fat levels and guar gum addition in mayonnaise-type emulsions on the sensory perception of smoke-curing flavour and salty taste," Food Quality and Preference, vol. 18, pp. 872-879, 2007.

[33] J. Lucas, V. Quintero, J. Vasco y L. Cuellar, "Evaluación de los parámetros de calidad durante la fritura de rebanadas de papa criolla," Scientia et Technica, vol. 16, nº48, pp. 299-304, 2011. 